\title{
Potential Hint of Dark Matter in Universe Maps
}

\section{A detected overlap of gravitational lenses and gamma-ray signals could shed light on the nature of dark matter.}

\section{by Francesca Calore*}

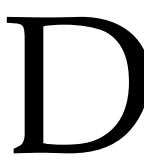
ark matter permeates the Universe, making up about $85 \%$ of its matter content. Astronomical and cosmological observations indicate that this elusive form of matter shaped the evolution of the Universe, leaving its imprint on the distribution of galaxies and large-scale cosmic structures. Now Simone Ammazzalorso at the University of Turin in Italy, Daniel Gruen at Stanford University in California, and colleagues have detected a signal that could allow scientists to shed light on

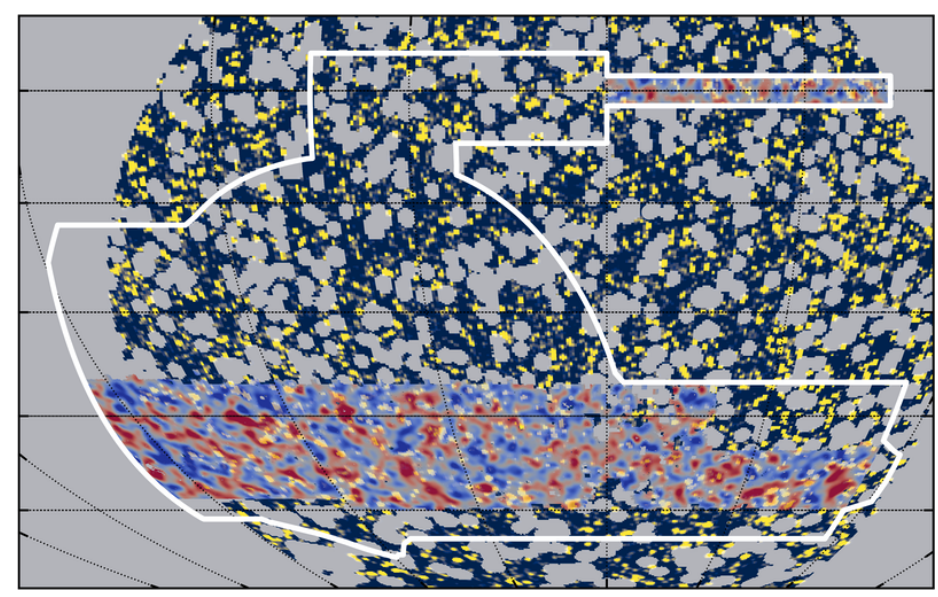

Figure 1: Researchers have discovered a correlation between the unresolved gamma-ray background and gravitational lensing. The gamma-ray data come from the Fermi Large Area Telescope (LAT) and the gravitational lensing data from the Dark Energy Survey (DES). The yellow color in the LAT image indicates regions of bright gamma-ray emission, while the red color in the DES map corresponds to regions of high matter density. (D.

Gruen/SLAC/Stanford; C. Chang/University of Chicago; A. Drlica-Wagner/Fermilab)

*French National Center for Scientific Research (CNRS) at the Annecy-le-Vieux Theoretical Physics Lab (LAPTh), Annecy-leVieux, France the nature of dark matter [1]. Matching observations made by the Dark Energy Survey (DES) and the Fermi Large Area Telescope (LAT), the team found a significant cross correlation between the positions of gravitational lenses, which are thought to trace dark matter, and those of gamma-ray photons, which are potentially emitted when dark matter self-destructs. Although their data indicates that the cross correlation predominantly comes from another class of astrophysical objects, the possibility that it partly arises from dark matter remains open.

The distribution of the Universe's matter is traditionally measured through gravitational lensing. This effect, predicted by Einstein, occurs when the path of light emitted by a distant object is distorted by the gravitational pull of matter between the object and Earth. Astronomers use gravitational lensing of galaxies to reconstruct maps, such as that created by the DES, of visible and dark matter [2].

While scientists know from these maps that dark matter exists, they don't yet know what form this matter takes. A prime candidate for dark matter is some new particle that weighs at least a few $\mathrm{GeV} / c^{2}$, where $1 \mathrm{GeV} / c^{2}$ is about the mass of a proton. Models predict that these dark matter particles should produce a flux of high-energy photons, known as gamma rays, when the particle density is large enough. Gamma-ray emission comes from the collision and subsequent annihilation of two dark matter particles and should be observable with current telescopes, such as the LAT [3]. Thus gamma-ray signals could act as dark matter tracers, with brighter signals indicating regions of space with higher concentrations of this elusive substance.

But dark matter isn't the only possible source of gamma rays. Gamma rays are also produced by more "mundane" astrophysical objects, such as by the remnants of exploding supernovae or by the jets launched by very active supermassive black holes, known as blazars. Some of these sources are either powerful enough or close enough to Earth for the LAT to detect. Most sources of gamma rays, however, are too faint to detect as individual objects and, instead, contribute to the cosmic gamma-ray "glow" obtained when the brightest sources are subtracted from observations made with gamma-ray telescopes. This leftover signal is dubbed the unresolved gamma-ray background [4]. Dark matter's 
gamma-ray emission could lie in this background, something that Ammazzalorso, Gruen, and colleagues explored with their new analysis [1].

In their study, the team cross correlated the DES map with that of the unresolved gamma-ray background, a process that involved searching for common patterns in the two datasets. The team found that the gamma-ray signal was brighter in regions that lensing shows contain more matter and dimmer where there is less. More specifically, at high gamma-ray energies and small angular separations $\left(<0.3^{\circ}\right)$, they detected a cross correlation with a signal-to-noise ratio greater than 5, qualifying the result a discovery. They also uncovered a hint of a cross correlation at larger angular scales, but with a lower statistical significance.

Positional coincidence of a bright gamma-ray spot with that of a dense region of dark matter density could hint at a common origin of the two signals [5]. But cross correlation alone is not enough to attribute the gamma rays to dark matter. For that, the team used gamma-ray emission models of astrophysical objects to look for the best match between the measured signal and the matter that might have produced it. Their modeling indicates that the cross correlation at small angular separations mostly arises from blazars. At larger angular scales, the models indicate that the gamma-ray signal could include an additional contribution from dark matter, with the statistical significance of this finding being about 3 sigma. This result is exciting as it marks one of the few hints at the existence of dark matter via indirect detection methods, and it opens up new possibilities for probing dark matter particle models. That said, current understanding of the physics of blazars is imperfect, and it may be that the large-scale cross correlation comes entirely from blazars. Either way, Ammazzalorso, Gruen, and colleagues unraveled something new, as there is a very low probability that the cross correlation signal they detected occurred by chance.

The new discovery is likely the first of many that will arrive with future observations. The DES plans to keep taking data and perform new analyses, and next-generation telescopes will provide more and better lensing data. The Legacy Survey of Space and Time (LSST) at the Vera Rubin Observatory in Chile is expected to start science operations at the end of 2022 and will map the history of several billion galaxies [6]. And the Euclid space telescope, currently being built by the European Space Agency and expected to launch in 2022, will explore the evolution of the Universe over the last 10 billion years, increasing the redshift horizon and the sensitivity to cosmic dark matter signals [7]. With deeper redshift coverage and a better angular resolution, future instruments will enable scientists to better understand the sources behind the Universe's gamma-ray glow and, potentially, uncover the nature of dark matter.

This research is published in Physical Review Letters.

\section{REFERENCES}

[1] S. Ammazzalorso et al., "Detection of cross-correlation between gravitational lensing and $\gamma$ rays," Phys. Rev. Lett. 124, 101102 (2020).

[2] J. Zuntz et al. (DES Collaboration), "Dark Energy Survey Year 1 results: Weak lensing shape catalogues," Mon. Not. Roy. Astron. Soc. 481, 1149 (2018).

[3] G. Bertone et al., "Particle dark matter: evidence, candidates and constraints," Phys. Rep. 405, 279 (2005).

[4] M. Fornasa and M. A. Sánchez-Conde, "The nature of the diffuse gamma-ray background," Phys. Rep. 598, 1 (2015).

[5] S. Camera et al., "A novel approach in the weakly interacting massive particle quest: cross-correlation of gamma-ray anisotropies and cosmic shear," Astrophys. J. 771, L5 (2013).

[6] https://www.lsst.org/.

[7] https://www.euclid-ec.org/.

10.1103/Physics. 13.31 